\title{
Electron Transport Properties of Graphene Device with Au Electrodes: A First-principles Investigation
}

\author{
Xiaotong Li, Yanwu Lu', Dan Li \\ Department of Physics, Beijing Jiaotong University, Beijing 100044, China \\ aemail: ywlu@bjtu.edu.cn
}

Keywords: Graphene; Electron Transport; Semiconductor Device; First-principles

\begin{abstract}
We present a first-principles investigation of electron transport in graphene contact with Au electrodes. Using destiny function theory and a non-equilibrium Green's function approach, we obtain the best transmission spectrum and current-voltage profiles for Au-graphene-Au contacting device. For this device, the current exist an obvious increase in scattering region as rotating graphene at a low bias voltage. The maximum current $(2.813 \mu \mathrm{A})$ is found at 30 degree of rotating graphene sheet. As the bias increase, there is a negative differential conductance effect at rotating 120 degree. These results provide some potential applications in oscillator and transistor devices.
\end{abstract}

\section{Introduction}

Graphene materials have attracted great interest for high-speed electronic device, because of its extreme high mobility and unique electronic structure $[1,2]$. Many researchers are now active in graphene research about semiconductor industry, planning graphene to be the candidate material. We mostly pay attention to using graphene in field-effect transistor (FET) and fabricating graphene on negative resistance effect oscillators [3]. For constructing graphene electronic device, we build $\mathrm{Au}$ electrode to contact graphene sheet. In such an Au-graphene-Au model, the electron transport property is associated with the contact resistance in scattering region[4]. In particular, the position of graphene and the edge-effect are also influence on electron transport processes [5, 6].

Graphene is a single layer of graphite with $\mathrm{sp}^{2}$ hybridized carbon atoms arranged in a honeycomb-like crystal structure. Since 2004, graphene has been fabricated successfully, and then it attracted a lot of attention of researchers. Because of the linear energy dispersion at Dirac points, graphene has a high-speed charge transfer properties, long-distance transmission characteristics of ballistic and zero carrier density limit conductivity [7]. These unique characteristics make graphene be ideal for the best in new generation of electronic devices.

In this paper, we provide a first-principles investigation of the electron transport of Au-graphene-Au nano-electronic device. As a zero-gap semiconductor, some electron transport properties in graphene need to be investigated for its potential applications. This study of Au-graphene-Au device gives a better theoretical model to get the electronic transmission spectrum, current-voltage characteristics and differential conductance.

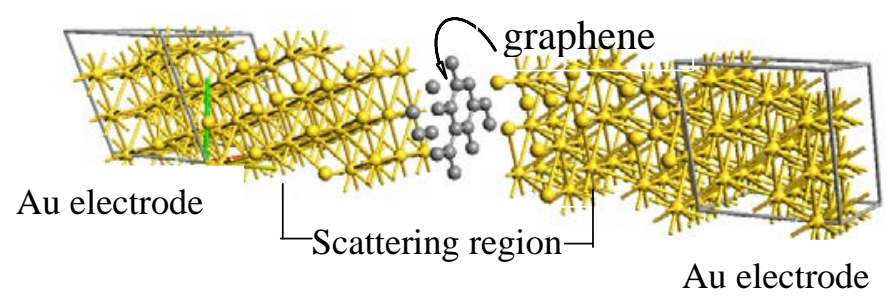

Fig.1. The model of graphene and Au Electrode Device: the yellow ones are gold atoms which are Au electrodes, the gray ones are graphene sheet which provides carriers for device. 


\section{Model and Computational Method}

In this paper, we studied the device consist of $\mathrm{Au}$ electrodes and graphene using the first-principles calculation. We use Au atoms as electrode and insert graphene in the middle of both electrodes. By rotating graphene's angle in the device, we analyzed the electron transport of the device under the different angles.

In Fig.1, this is the Au electrodes and graphene device model. In Au-electrode, the Miller indices of Au atoms are h k l (111) surface. We make a mirror operation at the $\mathrm{Z}=14 \AA$ A. At this position, $\mathrm{Au}$ atoms are repeated by 3 times along the $\mathrm{X}$ axis and the $\mathrm{Y}$ axis direction. With geometry optimization using Atomistix Toolkit (ATK) software, the optimum electrodes have been built. Electrode length: the left electrode length=7.06374 $\AA$, the right electrode length=7.06374 $\AA$, the scattering region length $=18.58165 \AA$, the vacuum region length $=8.9780 \AA$. Graphene sheet is put into the middle position ( $\mathrm{Z}=15.1773 \AA$ ) at the scattering region.

In this study, the building model and geometry optimizations have been performed by using the ATK based on density functional theory (DFT). The approach is an effective solution scheme of the first-principles investigation [8]. In our calculations, the electronic exchange-correlation energy is obtained under a local-density approximation (LDA). With ATK software package, we calculated the transmission spectrum for Au-graphene-Au device structure. From a non-equilibrium Green's function (NEGF) formalism, we can determine the transmission spectra for each base and various voltage biases with following formula:

$$
T(E, V)=\sum_{k} \sum_{n l} t_{n l}(E, k, V) t_{l n}^{+}(E, k, V),
$$

where $t(E, k, V)$ represents the transmission amplitude, which can be obtained from left electrode and right electrode wave functions [9]. By comparing several configurations, when the graphene layer is placed at the middle of the scattering region, the transmission spectrum of the device reach a optimal level [10]. Rotating the graphene sheet every 15 degree interval along $\mathrm{Z}$ axis, the transmission spectrum and I-V characteristics can be obtained from calculation results.

\section{Computational results and discussions}

In this study, the energy cut-off is set to 75 Hartree. The optimizations of the lattice constants and the atomic coordinates are made by the minimization of the total energy. The $3 \times 3$ supercell (containing 18 atoms) has been used to simulate the graphene sheet and the sheet placed in the middle of the two Au electrodes at $15.1773 \AA$ along $\mathrm{Z}$ axis. In practical calculations, the k-points mesh is $3 \times 3 \times 50\left(n_{a} n_{b} n_{c}\right)$. During all calculation processes, the transmission spectrum are obtained from energy ranges $-1 \mathrm{eV}$ to $1 \mathrm{eV}$ and the electron temperature is set $300 \mathrm{~K}$. In Fig.2, it shows curves of transmission coefficients as energy function. For the I-V characteristics, we applied the formula following:

$$
I(V)=\frac{2 e}{\hbar} \int_{-\infty}^{+\infty} T(E, V)\left[f\left(E-\mu_{L}\right)-f\left(E-\mu_{R}\right)\right] d E,
$$

where $f(E)$ is the Fermi distribution function. The bias voltage is defined as $V=\left(\mu_{L}-\mu_{R}\right) / e, \mu_{L}$ and $\mu_{R}$ are the Fermi level of the left and right electrodes, respectively. We calculated transmission spectrum under bias voltage $\mathrm{V}$ from $-1 \mathrm{~V}$ to $+1 \mathrm{~V}$ with interval $+0.2 \mathrm{~V}$. These calculations under geometry optimizations can be implemented with maximum force at $0.05 \mathrm{eV} / \AA$.

Figure 2 shows the transmission coefficients as a function of energy for the device with different configurations for rotating the graphene sheet. The maximum of transmission coefficient indicates that some electronic states at a certain energy exist the coherent effect, which corresponds to a local energy level $[11,12]$. The transmission coefficient of device at 0 and 180 degree overlaps each other. This means the zigzag edge and armchair edge of the graphene sheet are coincident in space, when we rotate it from the original place to 180 degree. For the curve with rotating 60 degree in 
Fig.2, there is a maximum of transmission coefficient peak at 0.2355 , which is near the Fermi level.

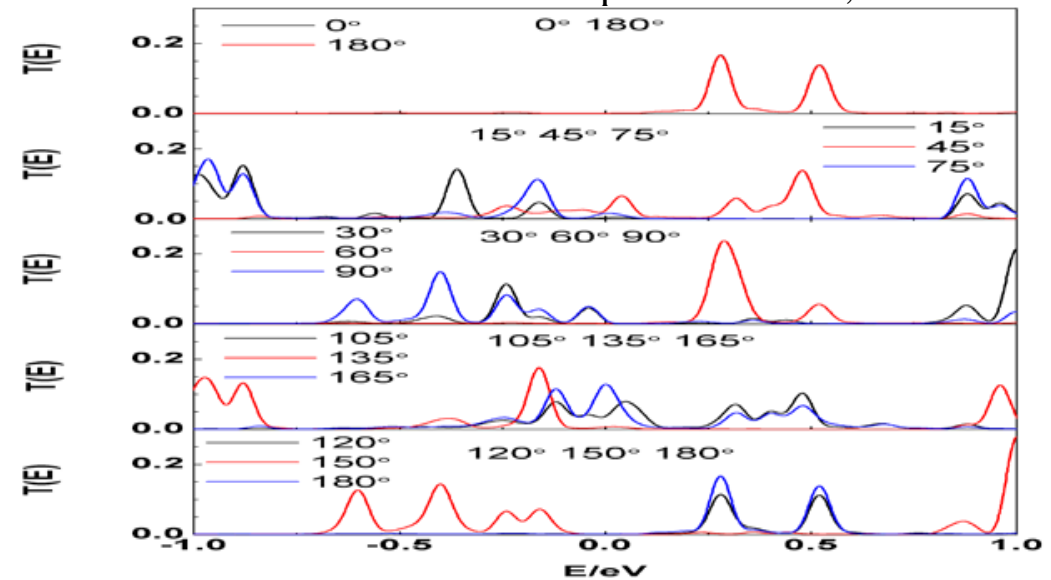

Fig.2. The transmission spectrum of device. For different rotating angles with 15 degree interval, these curves show the relation between transmission coefficient and energy.

We discuss the electric transport through Au-graphene-Au device. Figure 3 and Figure 4 display the current-voltage $(I-V)$ curves and conductance-voltage $(d I / d V-V)$ curves of Au-graphene-Au model with various rotating contact structures. In Fig.3, while the graphene sheet rotates at 30 degree angle, the maximum of the current of device is $2.813 \mu \mathrm{A}$ at $-1 \mathrm{~V}$ and the minimum of the current is $-1.569 \mu \mathrm{A}$ at $-0.73 \mathrm{~V}$. As $\mathrm{V}>-0.5 \mathrm{~V}$, the current is almost linear increasing with the bias voltage increasing, but as $-1 \mathrm{~V}<\mathrm{V}<-0.5 \mathrm{~V}$, the current decreases rapidly as the bias voltage increasing. In this case, the device has an obvious negative resistance effect [13].
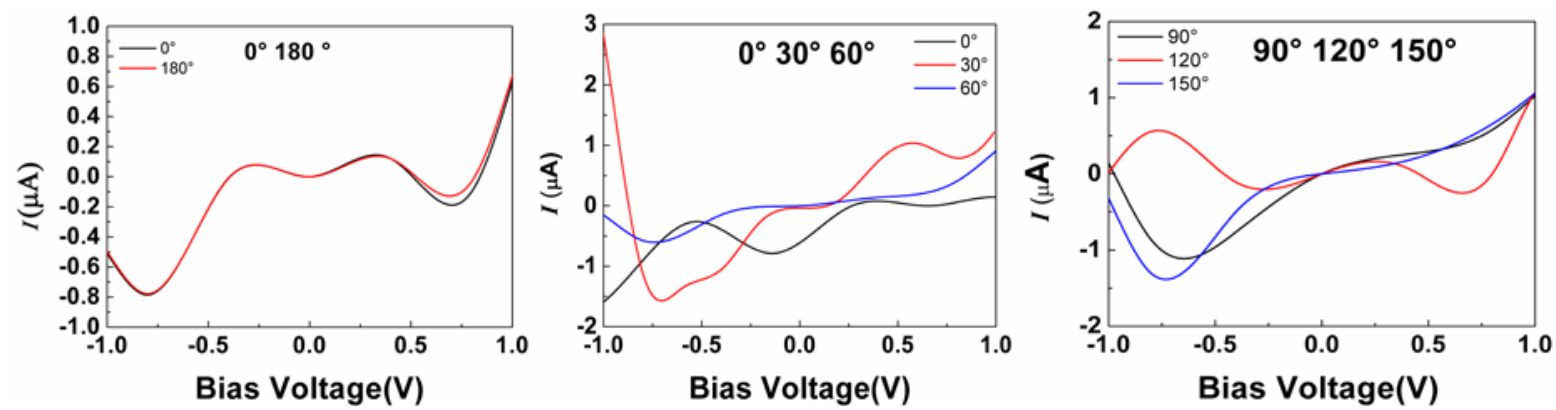

Fig.3.Current-voltage characteristics for the I-V picture of device based on Figure 2 transmission spectrum.

In Fig.4, there is negative differential conductancein every system, but the 120 angle system is the most significant.In particular, graphene rotating at 120 angle, $-1 \mathrm{~V}<\mathrm{V}<-0.5 \mathrm{~V}$, the differential conductance decreases from $4 \mu \mathrm{s}$ to $-2.5005 \mu \mathrm{s}$ with the bias voltage increasing. The maximum of the differential conductance is $5.923 \mu$ s.
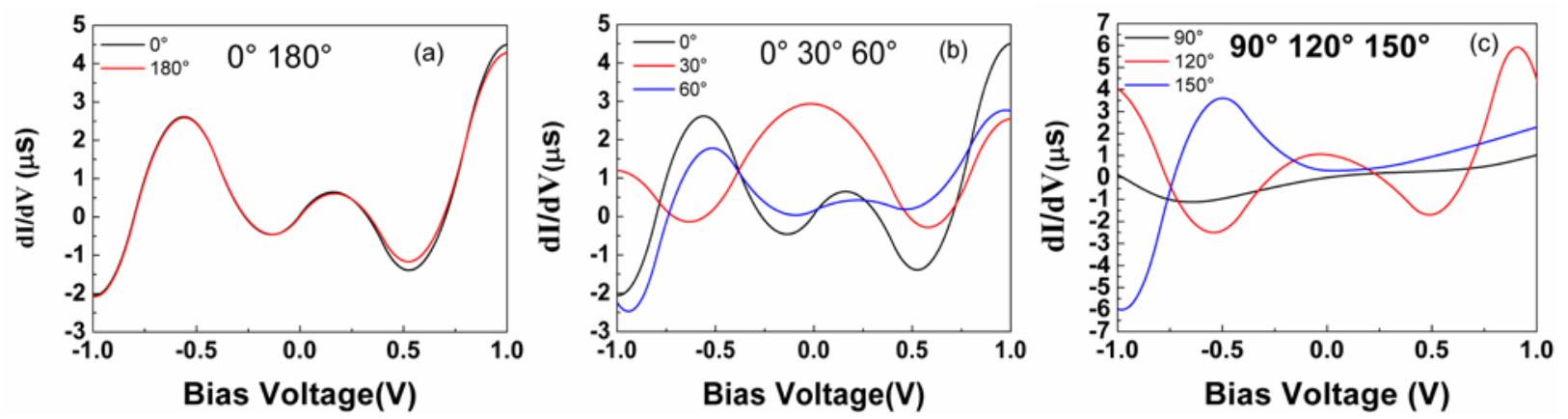

Fig.4. Differential conductance dI/dV of (a) $0^{\circ} / 180^{\circ}$ (b) $0^{\circ} / 30^{\circ} / 60^{\circ}$ and (c) $90^{\circ} / 120^{\circ} / 150^{\circ}$ 


\section{Conclusion}

We investigate electron transport across the Au-graphene-Au device using ATK software under DFT frame. Our results show that the contact region between graphene and Au electrodes has different influences by rotating graphene sheet. The current and conductance of Au-graphene-Au device depend obviously on rotating angle with the changing range of $-1.57 \mu \mathrm{A}$ to $2.81 \mu \mathrm{A}$ and $-22.80 \mu \mathrm{S}-38.42 \mu \mathrm{S}$, respectively, for eight different contact structures. This feature is largely originated from electronic state coherent effects, which correspond to transmission coefficient peaks. It is obvious that a negative differential conductance appears in the Au-graphene-Au model, which observed the current decreasing as the bias voltage increasing. In this case, the transmission curves indicate that the charge transferring from $\mathrm{Au}$ electrode to graphene plays an important role in electron transport of electrode-graphene-electrode, whereas electric field scattering and edge effect of graphene have also influence on the transmission enhancement of this device.

\section{Acknowledgement}

This work was supported by NSFC (Grant No.60976070).

\section{References}

[1] Frank Schwierz. Graphene transistors [J]. Nature nanotechnology, 2010, 5: 487-496.

[2] Xiang Ji, Jinyu Zhang, Yan Wang,He Qian and Zhiping Yu. A theoretical model for metal/graphene contact resistance using DFTNEGF method [J]. Phys.Chem.Chem. Phys, 2013, 15: 17883-17886.

[3] Joshua A. Robinson, Michael LaBella, Mike Zhu, Matt Hollander, Richard Kasarda, Zachary Hughes,Kathleen Trumbull, Randal Cavalero, and David Snyder. Contacting graphene [J]. Appl. Phys. Lett, 2011, 98: 053103.

[4] David K.Ferry.StephenM.Goodnick and Jonathan Bird. Transport in nanostructure second edition [M]. Cambridge University Press, 2009

[5] Pekka Koskinen, Sami Malola, and Hannu Häkkinen. Evidence for Graphene Edges Beyond Zigzag and Armchair [J].Phys.Rev.B, 2009, 80(7): 073401.

[6] Frank J. Owens. Electronic and magnetic properties of armchair and zigzag graphene nanoribbons [J]. J. Chem. Phys, 2008, 128: 194701.

[7] D. Bischoff, A. Varlet, P. Simonet, M. Eich, H. C. Overweg, T. Ihn, and K. Ensslin. Localized charge carriers in graphene nanodevices. [J]. Appl. Phys. Rev, 2015, 2: 031301.

[8] Rui Mao, Byoung Don Kong, and Ki Wook Kim. Thermal Transport properties of metal/MoS2 interfaces from first principles [J]. J. Appl. Phys, 2014, 116: 034302.

[9]Hannah L. McFarland, Towfiq Ahmed, Jian-Xin Zhu,AlexanderV.Balatsky,and Jason T. Haraldsen. First-Principles Investigation of Nanopore Sequencing Using VariableVoltage Bias on Graphene-Based Nanoribbons [J]. J. Phys. Chem. Lett, 2015, 6(13): 2616-2621.

[10] Frederico D. Novaes, Riccardo Rurali,and Pablo Ordejón. Electronic Transport between Graphene Layers Covalently Connected by Carbon Nanotubes [J]. ACS Nano, 2010, 4(12): 7596-7602.

[11] Chengyu Yanga, Quanfang Chena. Electronic structure and transport properties of carbon nanotube adsorbed with a copper chain [J]. International Journal of Smart and Nano Materials, 2013, 4(3): 143-149. 
[12]Hongmei Liu, Hisashi Kondo, and TakahisaOhno. Effect of contact area on electron transport through graphene-metal interface [J]. J.Chem.Phys, 2013, 139: 074703

[13] WanzhiQiu, Phuong Duc Nguyen, and EfstratiosSkafidas. Graphene nanopores as negative differential resistance devices [J]. J.Appl.Phys, 2015, 117: 054306 